\title{
Darwin-Foldy term and proton charge radius
}

\author{
M. Bawin ${ }^{\mathrm{a}}$ and S. A. Coon ${ }^{\mathrm{b}}$ \\ ${ }^{a}$ Université de Liège, Institut de Physique B5, Sart Tilman, 4000 Liège 1, Belgium \\ ${ }^{\text {b} P h y s i c s ~ D e p a r t m e n t, ~ N e w ~ M e x i c o ~ S t a t e ~ U n i v e r s i t y, ~ L a s ~ C r u c e s, ~ N M ~ 88003, ~ U S A ~}$
}

Back in 1958 Foldy [1], following Pauli's early work on incorporating the anomalous magnetic moment of a particle into the Dirac equation, proposed a systematic way of including finite size effects in the description of a relativistic spin $\frac{1}{2}$ particle in interaction with an external electromagnetic field by means of the Dirac equation. An apparently perturbing feature of Foldy's treatment when applied to a neutron is that the magnitude of the neutron charge radius is accounted for almost completely by treating the neutron as a point Dirac particle (the "Darwin-Foldy"term), thus apparently leaving no room for finite size effects [2] but rather a so-called "intrinsic radius" much smaller than the Compton wavelength of the neutron.

We recently suggested that the composite nature of the neutron can be described by including Dirac-Pauli form factors in the Dirac equation. Then, the Darwin-Foldy term is cancelled exactly by a term in a low-momentum expansion of the form factors, leaving the quantity measured in neutron-electron scattering to be directly identified with the neutron charge radius [3]. Our result agrees with recent results by Isgur [4] (in the context of the valence quark model) and Cardarelli and Simula [5] (in the relativistic constituent quark model). Note however that the interpretation of the Darwin-Foldy term for a particle with structure remains a controversial topic [6]. For example, one can read in [6] that the intrinsic radius "provides a measure of the failure of the Dirac-Pauli equation to account in detail for the observed properties of the neutron at low energy".

In this contribution we study the Dirac equation for a finite size proton in an external electric field with explicit introduction of Dirac-Pauli form factors. Our aim is twofold. On the one hand, we wish to study whether our conclusions regarding the exact cancellation between Dirac form factor and Foldy term contributions occurring for the neutron still hold for the proton. On the other hand, we wish to clearly illustrate some of the specific features of the description of a composite particle like the proton with the Dirac equation. Indeed, the Dirac equation includes the effect of virtual particle-antiparticle creation pair terms (Foldy's "dancing motion") which endow even a charged point particle with an effective size, the Darwin term. (The "Darwin-Foldy" term of the neutron, however, arises from the large anomalous magnetic moment of this neutral particle). Whether such an effect should be included in the definition of the particle charge radius is at the heart of the controversy surrounding the Dirac approach description of the nucleon charge radius.

Our main results are the following:

1) The Dirac form factor contribution continues to cancel the Darwin-Foldy contribution 
from the proton's anomalous magnetic moment (as was also demonstrated in the model of Ref. [0]). There remains, however, the standard Darwin term (the $\frac{e}{8 m^{2}}$ term) coming from the normal part of the proton magnetic moment, a consequence of the Zitterbewegung of the charged Dirac particle.

2) The above cancellation crucially depends upon using the Dirac equation with form factors to describe the finite size and anomalous magnetic moment of the baryons. The resulting charge radius is then that of the Sach's form factor [3] and is thus the same as the definitions used in recent chiral perturbation theory calculations [10].

Our starting point is the Dirac Hamiltonian $\mathrm{H}$ for a finite size proton in an external field with explicit introduction of Dirac-Pauli form factors :

$$
H=\boldsymbol{\alpha} \cdot \mathbf{p}+\beta m+i \frac{e F_{2}}{2 m} \beta \boldsymbol{\alpha} \cdot \mathbf{E}+e F_{1} V
$$

where $F_{1}$ and $F_{2}$ are, respectively, the proton Dirac and Pauli form factors, and $V$ is the potential associated with the external field $\mathbf{E}$ in the Coulomb gauge. The connection between $F_{1}, F_{2}$, and the electric and magnetic Sachs form factors $G_{E}$ and $G_{M}$ is:

$$
\begin{aligned}
G_{E}(t) & =F_{1}(t)+\frac{t}{4 m^{2}} F_{2}(t) \\
G_{M}(t) & =F_{1}(t)+F_{2}(t)
\end{aligned}
$$

The Sachs form factors have simple interpretations as the spatial Fourier transforms of the nucleon's charge and magnetization distributions in the Breit frame[9], where $t=-\mathbf{q}^{2}$, and $\mathbf{q}$ is the usual 3-dimensional momentum transfer. Specifically, we have:

$$
\rho_{P}(\mathbf{r})=\left(\frac{1}{2 \pi}\right)^{3} \int d^{3} \mathbf{q} e^{+i \mathbf{q} \cdot \mathbf{r}} G_{E}\left(-\mathbf{q}^{2}\right)
$$

such that the normalization $\int d^{3} \mathbf{r} \rho_{P}(\mathbf{r})$ is one for the proton. One defines the square of the proton charge radius $r_{E p}^{2}$ by the relation:

$$
G_{E}\left(-\mathbf{q}^{2}\right)=1-\frac{1}{6} r_{E p}^{2} \mathbf{q}^{2}+\cdots
$$

Taking into account the relations [3]:

$$
\begin{aligned}
& F_{1}(0)=1 \\
& F_{1}^{\prime}(0) \equiv \frac{\partial F_{1}\left(\mathbf{q}^{2}=0\right)}{\partial \mathbf{q}^{2}}=-\frac{1}{6} r_{E p}^{2}+\frac{\kappa}{4 m^{2}},
\end{aligned}
$$

where $\kappa$ is the proton anomalous dimensionless magnetic moment, we eventually get the following equation from the Foldy-Wouthuysen reduction of the Dirac Hamiltonian (1) together with a low $\mathbf{q}^{2}$ expansion of $\tilde{V}(\mathbf{q})=e F_{1}\left(-\mathbf{q}^{2}\right) V(\mathbf{q})$ [3]:

$$
\begin{aligned}
\left\{\frac{\mathbf{p}^{2}}{2 m}-\frac{e \mathbf{p}}{2 m^{2}} \cdot(\mathbf{E} \times \boldsymbol{\kappa})+\frac{e^{2} \kappa^{2} \mathbf{E}^{2}}{8 m^{3}}-e\left(\frac{r_{E p}^{2}}{6}\right.\right. & \left.+\frac{1}{8 m^{2}}\right)(\boldsymbol{\nabla} \cdot \mathbf{E}) \\
& \left.+V+\frac{\boldsymbol{\nabla}^{2}}{8 m^{2}} \tilde{V}\right\} \psi=E \psi,
\end{aligned}
$$


where $\tilde{V}$ is given by:

$$
\tilde{V}(\mathbf{r}) \equiv e F_{1} V=e\left(\frac{1}{2 \pi}\right)^{3} \int d^{3} \mathbf{q} e^{i \mathbf{q} \cdot \mathbf{r}} F_{1}\left(-\mathbf{q}^{2}\right) V(\mathbf{q}) .
$$

In equation (7), the Dirac form factor contribution continues to cancel the Darwin-Foldy contribution from the proton's anomalous magnetic moment (as was also demonstrated in the model of Ref. [5]). There remains, however, the standard Darwin term (the $\frac{e}{8 m^{2}}$

term) coming from the normal part of the proton magnetic moment, a consequence of the dancing motion of the charged Dirac particle. By convention, that term, also present for a structureless electron, does not contribute to the charge radius. Thus the only structure term in the coefficient of the external field charge density is the physical charge radius square $r_{E p}$. We therefore conclude that the inclusion of the nucleon form factor together with the definition of the nucleon charge radius given by equation (4) removes the apparent difficulties associated with using the Dirac equation to describe the nucleon charge radius. Contrary to previous statements in the literature (匹11), the Dirac equation does not necessarily fail at the level of the Darwin-Foldy term.

\section{REFERENCES}

1. L. L. Foldy, Rev. Mod. Phys. 30, 471(1958); see also L. L. Foldy, Phys. Rev. 87, $688(1952)$.

2. L. Ya. Glozman and D. O. Riska, Phys. Lett. B459 , 49(1999).

3. M. Bawin and S. A. Coon, Phys. Rev. C60 , 025207-1(1999).

4. N. Isgur, Phys. Rev. Lett. 83, 272(1999).

5. F. Cardarelli and S. Simula, Phys. Lett. B467 , 1(1999).

6. J. Byrne, Neutron News, 5, no. 4, 15(1994).

7. Yu. A. Alexandrov, Neutron News, 5, no. 1, 20(1994); Neutron News, 5, no. 4, 17(1994); Phys. Part. and Nuc. 30, 29(1999).

8. G. G. Bunatian, V. G. Nikolenko, A. B. Popov, G. S. Samosvat and T. Yu. Tretyakova, Z. Phys. A359, 337(1997).

9. R. G. Sachs, Phys. Rev. 126, 2256(1962).

10. S. J. Puglia, M. J. Ramsey-Musolf and Shi-Lin Zhu, hep-ph/0008140. The "Foldy" term of B.Kubis and Ulf-G.Meissner, hep-ph/0007056 and of this reference is our "Darwin-Foldy" term.

11. J. Achtzehnter and L. Wilets, Phys. Rev. C38, 5(1988). 